\title{
A Selected Library of Transport Coefficients for Combustion and Plasma Physics Applications
}

\author{
L.D. Cloutman
}

\section{August 1, 2000}

Lawrence

Livermore

National

Laboratory 
Lawrence Livermore National Laboratory report

UCRL-ID-139893

August 2000

\title{
A SELECTED LIBRARY OF TRANSPORT COEFFICIENTS FOR COMBUSTION AND PLASMA PHYSICS APPLICATIONS
}

\author{
Lawrence D. Cloutman
}

\begin{abstract}
COYOTE and similar combustion programs based on the multicomponent NavierStokes equations require the mixture viscosity, thermal conductivity, and species transport coefficients as input. This report documents a model of these molecular transport coefficients that is simpler than the general theory, but which provides adequate accuracy for many purposes. This model leads to a computationally convenient, selfcontained, and easy-to-use source of such data in a format suitable for use by such programs. We present the data for various neutral species in two forms. The first form is a simple functional fit to the transport coefficients. The second form is the use of tabulated Lennard-Jones parameters in simple theoretical expressions for the gas-phase transport coefficients. The model then is extended to the case of a twotemperature plasma. Lennard-Jones parameters are given for a number of chemical species of interest in combustion research.
\end{abstract}




\section{Introduction}

Viscosity, thermal conductivity, and species transport coefficients are an important part of any effort to make realistic numerical simulations of reactive flows, especially in the laminar regime. Inclusion of molecular transport effects in a computational fluid dynamies (CFD) code requires resolution of three issues. The first is the level of approximation used in computing the molecular diffusion fluxes of mass, momentum, and energy. The second is the specification of the appropriate transport coefficients for each species. The third is the development of numerical algorithms for implementation into CFD codes. We shall address only the first two issues here:

The full theory of molecular transport in multicomponent fluids is extremely complex ([1], for example) and is computationally unsuited for use in a multidimensional CFD program. It is necessary to simplify the problem by introducing some level of approximation. Coffee and Heimerl [2] present a study comparing five such models. The most sophisticated is described also by Dixon-Lewis [3], and it requires solution of two linear systems in order to compute the mass and heat fluxes at a single point in space and time. This model has been implemented in the widely used CHEMKIN program [4]-[7], but it is rather computationally intensive for use in multidimensional CFD codes. A simpler approximation is adopted that avoids solving numerous linear systems and is discussed by Coffee and Heimerl and by Paul [8].

The second part of the problem is the specification of the species transport cocfficients. Unfortunately, the available data are scattered widely throughout the literature of chemistry, physics, and engincering in a wide variety of formats and units. This information must be collected, assessed for accuracy, and put into an appropriate format before it can be used in any particular CFD program. Due to the large number of species of potential interest in combustion studics (potentially a thousand or more for a single problem), creating and maintaining such a database can become a major task. The program system CHEMKIN [4][7] is an excellent example of a computerized database designed to address this need. Use of this package has two advantages: avoidance of the effort required to independently collect the data in a usable form and the ability to use the same gas physics as a wide community 
of researchers. For these reasons, sclected parts of the CHEMKIN transport model were adopted, most notably the use of the Lennard-Jones model for most neutral specics.

The remainder of this report presents the molecular transport model used in the COYOTE reactive-flow CFD program [9]. This includes both the constitutive relations for the mass, momentum, and energy fluxes as well as expressions for the transport coefficients. Section 2 presents this information for un-ionized gases, including two models for computation of species viscosities and thermal conductivities. These two choices are a simple parametric fitting function (a slightly generalized Sutherland equation) and the use of the Lennard-Jones parameters in simple analytical expressions for the transport coefficients. Sample data for both approaches are given in the tables in this report. Binary diffusion coefficients are computed from the Lennard-Jones model. Section 3 presents an extension of this model to ionized gases. In all cases, simple mixture rules are used to get viscosity and conductivity for a mixture with an arbitrary composition. The final section contains some concluding comments.

\section{Transport Coefficients for Un-Ionized Gases}

Computing the transport cocfficients for a multicomponent fluid in complete generality is a complex, difficult task. We adopt approximations that are computationally tractable. In particular, we want to avoid the linear sytems used in CHEMKIN while retaining as much similarity as possible. In this section, we discuss momentum, energy, and mass transport in separate subsections. Models for the transport coefficients and for the various fluxes are presented in the next three subsections.

\subsection{The Coefficient of Viscosity}

Let $\mathrm{T}$ be the viscous stress tensor for a Newtonian fluid,

$$
\begin{gathered}
\mathrm{T}=\mu\left[\nabla \mathbf{u}+(\nabla \mathbf{u})^{T}\right]+\mu_{1}(\nabla \cdot \mathbf{u}) \mathbf{U} \\
=\mu\left[\nabla \mathbf{u}+(\nabla \mathbf{u})^{T}\right]+\left(\mu_{b}-\frac{2 \mu}{3}\right)(\nabla \cdot \mathbf{u}) \mathbf{U} .
\end{gathered}
$$

Here $\mathbf{u}$ is the velocity of the fluid mixture, $\mathrm{U}$ is the unit tensor, $\mu$ is the cocfficient of viscosity, $\mu_{1}$ is the second coefficient of viscosity, and $\mu_{b}$ is the bulk viscosity. 
What we require is a procedure for spccifying the coefficient of viscosity for the mixture of species composing the fluid. The first step in calculating the mixture viscosity is to cvaluate the viscosity for cach species. At the pressures and temperatures found in typical combustion systems, the species coefficients of viscosity are almost independent of density, so we assume they are functions only of temperature. We allow two ways of specifying the coefficient of viscosity. The first is a simple four-parameter analytic function that is suitable for fitting tabulated viscosity data. The second form is based on the Lennard-Jones parameters for each gas species.

\begin{tabular}{lllrrrr}
\multicolumn{7}{c}{ Table 1. Coefficient of Viscosity } \\
Species & $n_{\alpha}$ & $A_{\alpha}$ & $B_{\alpha}$ & $C_{\alpha}$ & $T$ Range & Ref. \\
Air & 1.5 & $1.457 \times 10^{-5}$ & 110.0 & 0.0 & Unknown & - \\
Air & 1.51 & $1.387 \times 10^{-5}$ & 105.1 & 0.0 & $79-1407$ & {$[13]$} \\
$\mathrm{N}_{2}$ & 1.52 & $1.186 \times 10^{-5}$ & 86.54 & 0.0 & $251-1098$ & {$[13]$} \\
$\mathrm{O}_{2}$ & 1.46 & $2.294 \times 10^{-5}$ & 164.4 & 0.0 & $273-1102$ & {$[13]$} \\
$\mathrm{O}_{2}$ & 1.5 & $1.16 \times 10^{-5}$ & 0.0 & 0.0 & - & {$[14]$} \\
$\mathrm{C}_{3} \mathrm{H}_{8}$ & 1.72 & $1.802 \times 10^{-6}$ & 101.0 & 0.0 & $290-472$ & {$[13]$} \\
$\mathrm{SF}_{6}$ & 1.7 & $3.526 \times 10^{-6}$ & 73.1 & 0.0 & $223-573$ & {$[15]$} \\
$\mathrm{H}_{2}$ & 1.67 & $1.963 \times 10^{-6}$ & 2.187 & 0.0 & $89-1098$ & {$[13]$} \\
$\mathrm{H}$ & 1.5 & $3.95 \times 10^{-6}$ & 0.0 & 0.0 & - & {$[14]$} \\
$\mathrm{O}$ & 1.5 & $1.15 \times 10^{-5}$ & 0.0 & 0.0 & - & {$[14]$} \\
$\mathrm{OH}$ & 1.5 & $1.10 \times 10^{-5}$ & 0.0 & 0.0 & - & {$[14]$} \\
$\mathrm{H}_{2} \mathrm{O}$ & 1.5 & $1.60 \times 10^{-5}$ & 0.0 & 0.0 & - & {$[14]$} \\
$\mathrm{HO}_{2}$ & 1.5 & $1.16 \times 10^{-5}$ & 0.0 & 0.0 & - & {$[14]$} \\
$\mathrm{H}_{2} \mathrm{O}_{2}$ & 1.5 & $1.16 \times 10^{-5}$ & 0.0 & 0.0 & - & {$[14]$} \\
$\mathrm{He}$ & 1.695 & $3.614 \times 10^{-6}$ & -9.549 & 0.0 & $81-1090$ & {$[13]$}
\end{tabular}

The first approach is based on a fitting function that takes advantage of the fact that simple kinetic theory predicts that the viscosity of a gas is proportional to $T^{1 / 2}$, where $T$ is the absolute temperature. We adopt a slightly more complex parameterization, a gencralized Sutherland formula, as our fitting function:

$$
\mu_{\alpha}=\begin{aligned}
& A_{\alpha} T^{n_{\alpha}} \\
& B_{\alpha}+T
\end{aligned}+C_{\alpha}
$$

where $\alpha$ denotes the particular species, and $A_{\alpha}, B_{\alpha}, C_{\alpha}$, and $n_{\alpha}$ are constants. Values of the constants are given in Table 1 for a limited selection of species. The units are all cgs, that 
is poise $(\mathrm{g} / \mathrm{cm}-\mathrm{s})$ for the viscosity and kelvins (K)for $T$. To convert to SI units, multiply the cgs viscosity by 0.1 . The constants in Table 1 were evaluated by setting $C_{\alpha}=0.0$ and fitting the viscosity data in the references at three temperatures (usually at the extremes and middle of the temperature range provided). The constant $C_{\alpha}$ is provided in the code mostly as a convenience in setting the viscosity to a constant value in the CFD code for certain numerical experiments, although it could also have been used to fit a fourth data point.

The accuracy of the fits varies somewhat. Most of the fits in Table 1 have crrors of at most a few percent in the temperature range given in the table, and they are often better than one percent over wide temperature ranges. Extrapolation beyond the given temperature range is always risky, although much less so on the high temperature end. Strong non-ideal gas effects can occur at low temperature that are not accounted for in the fits. For example, the He fit is approximately a factor of two in error around $20 \mathrm{~K}$, and it gets worse rapidly as the singularity at $9.549 \mathrm{~K}$ is approached.

The second approach uses the Lennard-Jones parameters to estimate the viscosity (in cgs units),

$$
\mu_{\alpha}=\frac{5}{16}\left(\frac{m_{\mathrm{H}} k_{\mathrm{B}}}{\pi}\right)^{1 / 2} \frac{\left(M_{\alpha} T\right)^{1 / 2}}{\sigma_{\alpha}^{2} \Omega^{(2,2) *}\left(T_{\alpha}^{*}\right)}=2.6693 \times 10^{-5} \frac{\left(M_{\alpha} T\right)^{1 / 2}}{\sigma_{\alpha}^{2} \Omega^{(2,2) *}\left(T_{\alpha}^{*}\right)},
$$

where $M_{\alpha}$ is the molecular weight, $m_{\mathrm{H}}$ is the mass of one atomic mass unit in grams, $k_{\mathrm{B}}$ is the Boltzmann constant, $\sigma_{\alpha}$ is the collision diameter in $\AA$, and $\Omega^{(2,2) *}\left(T_{\alpha}^{*}\right)$ is the collision integral approximated by

$$
\Omega^{(2,2) *}\left(T_{\alpha}^{*}\right)=1.147\left(T_{\alpha}^{*}\right)^{-0.145}+\left(T_{\alpha}^{*}+0.5\right)^{-2},
$$

where $T_{\alpha}^{*}=T / T_{e \alpha}=T k_{\mathrm{B}} / \epsilon_{\alpha}$ is the reduced temperature and $\epsilon_{\alpha}$ is the Lennard-Jones potential well depth $[10,11]$. Kee, et al. [5] recommend interpolation in Table $\mathrm{V}$ of Monchick and Mason [12], which includes dependence of the collision integral on the reduced dipole moment of the molecule, $\delta$, as well as dependence on $T_{e \alpha}$. Equation 4 is accurate to a few percent for Monchick and Mason's $\delta<0.5$ at low temperatures, and it becomes valid for larger values of $\delta$ at higher temperatures. Table 3 gives values of $\sigma_{\alpha}$ and $\epsilon_{\alpha} / K_{B}$ for selected species. 
Once the species viscosities have been calculated, they must be combined to provide the viscosity of the fluid mixture. We adopt Wilke's law [13] (see also Bird et al. [14]). For $N$ species,

$$
\mu=\sum_{\alpha=1}^{N} \underset{\sum_{\beta=1}^{N} X_{\beta} \mu_{\alpha \beta}}{X_{\alpha \beta}}
$$

where $X_{\alpha}$ is the mole fraction of species $\alpha$ and where

$$
\Phi_{\alpha, \beta}=8^{-1 / 2}\left(1+\frac{M_{\alpha}}{M_{\beta}}\right)^{-1 / 2}\left[1+\left(\frac{\mu_{\alpha}}{\mu_{\beta}}\right)^{1 / 2}\left(\frac{M_{\beta}}{M_{\alpha}}\right)^{1 / 4}\right]^{2} .
$$

In almost all studies, the bulk viscosity is set to zero, which is correct, strictly speaking, only for perfect monatomic gases. Thompson [16] gives a short table of bulk viscosities at a pressure of one atmosphere and a temperature of $300 \mathrm{~K}$. The ration $\mu_{b} / \mu$ is 3.2 for $\mathrm{H}_{2}$, 0.8 for $\mathrm{N}_{2}, 0.4$ for $\mathrm{O}_{2}, 1000$ for $\mathrm{CO}_{2}$, and 0.6 for air. Unpublished COYOTE simulations of a steady, one-dimensional stoichiometric laminar ethylene-air flame with a reduced (17 species, 32 reactions) kinctics mechanism showed no significant effect of $\mu_{b}$ on cither the flame speed or structure, even though $\mathrm{CO}_{2}$ was several percent of the reaction products. This is not surprising even though the velocity divergence is quite large in the flame front. The velocity field is sufficiently smooth even in the flame front that the viscous terms have very little effect on the dynamics compared to the heat release.

\subsection{Thermal Conductivities}

The heat flux is a complicated function, and COYOTE uses

$$
\mathbf{q}=-K \nabla T+\sum_{\alpha} h_{\alpha}(T) \mathbf{J}_{\alpha}-\sum_{\alpha} \frac{R T}{M_{\alpha} X_{\alpha}} D_{\alpha}^{T} \mathbf{d}_{\alpha}
$$

where $R$ is the gas constant, $K$ is the multicomponent thermal conductivity, $h_{\alpha}$ is the specific enthalpy of species $\alpha, D_{\alpha}^{T}$ is the multicomponent thermal diffusion coefficient, and

$$
\mathbf{d}_{\alpha}=\nabla X_{\alpha}+\left(X_{\alpha}-Y_{\alpha}\right) \frac{\nabla P}{P}-\frac{1}{P}\left[\rho_{\alpha} \mathbf{F}_{\alpha}-Y_{\alpha} \sum_{\beta} \rho_{\beta} \mathrm{F}_{\beta}\right],
$$

where $Y_{\alpha}$ is the species mass fraction $\rho_{\alpha} / \rho$ and $\mathrm{F}_{\alpha}$ is the body force per unit mass (acceleration) acting on species $\alpha[1,5]$. Calculation of the species mass fluxes and thermal diffusion cocfficients will be considered in the next section. 
In the original COYOTE program, $K$ was calculated from the mixture viscosity $\mu$ and a constant mixture Prandtl number. This capability has been expanded to allow calculation of the conductivity based on the local composition and temperature, just as was done for the viscosity. One option is to fit species conductivity data using the same functional form as for the viscosity (that is, equation 2). Results of a few fits are given in Table 2.

Table 2. Species Thermal Conductivities

$\begin{array}{lllllll}\text { Species } & n_{\alpha} & A_{\alpha} & B_{\alpha} & C_{\alpha} & T \text { Range } & \text { Ref. } \\ \text { Air } & 1.5 & 2.255 \times 10^{2} & 150.0 & 0.0 & 233-322 & {[17]} \\ \mathrm{He} & 1.63 & 4.167 \times 10^{2} & 2.635 & 0.0 & 33-322 & {[17]} \\ \mathrm{N}_{2} & 1.51 & 1.935 \times 10^{2} & 110.4 & 0.0 & 88-322 & {[17]} \\ \mathrm{O}_{2} & 1.86 & 2.126 \times 10^{1} & 22.26 & 0.0 & 88-366 & {[17]} \\ \mathrm{C}_{3} \mathrm{H}_{8} & 2.69 & 1.167 \times 10^{-1} & 2.409 & 0.0 & 233-366 & {[17]} \\ \mathrm{SF}_{6} & 1.8 & 2.296 \times 10^{1} & 140.5 & 0.0 & 300-4000 & {[15]} \\ \mathrm{H}_{2} & 1.5 & 1.522 \times 10^{3} & 124.4 & 0.0 & 88-322 & {[17]} \\ \mathrm{H} & 1.5 & 1.173 \times 10^{3} & 0.0 & 0.0 & - & {[18]} \\ \mathrm{O} & 1.5 & 2.134 \times 10^{2} & 0.0 & 0.0 & - & {[18]} \\ \mathrm{OH} & 1.5 & 2.69 \times 10^{2} & 0.0 & 0.0 & - & {[18]} \\ \mathrm{H}_{2} \mathrm{O} & 1.5 & 4.596 \times 10^{2} & 1036.2 & 0.0 & 255-367 & {[17]}\end{array}$

The sccond option is based on the Lennard-Jones parameters. Following the procedure of Hayashi and Hishida [10], we calculate the conductivity from the viscosity using the Eucken correction, which is discussed also by Forziger and Kaper [19] and by Hirschfelder, Curtis, and Bird [1]:

$$
K_{\alpha}=0.25\left(9 \gamma_{\alpha}-5\right) \mu_{\alpha} C_{\mathrm{v} \alpha}
$$

where $C_{\mathrm{v} \alpha}$ is the specific heat at constant volume and $\gamma_{\alpha}$ is the ratio of specific heats. Kee, et al. [5] describe a more complex approximation that we have not implemented. Their model accounts more accuratcly for the internal quantum states of the molecules.

There are two possible mixture rules for the conductivity. The first is to use equations 5 and 6 with $\mu_{\alpha}$ replaced by $K_{\alpha}$, but with the same values of $\Phi_{\alpha \beta}$ as used for the mixture viscosity [14]. The second rule is due to Mathur et al. [20] and is recommended by Kee, et al. [5]:

$$
K=\frac{1}{2}\left[\sum_{\alpha=1}^{N} X_{\alpha} K_{\alpha}+\left(\sum_{\alpha=1}^{N} X_{\alpha} / K_{\alpha}\right)^{-1}\right]
$$


We presently use the former rule in COYOTE. Paul [8] recommends against the latter rule and recommends instead a slightly modified version of equations 5 and 6 . Mixture rules are discussed also by Brokaw [21].

\subsection{Binary Diffusion Coefficients}

Calculation of the exact diffusional mass fluxes is an extremely complicated task (for example, [1], [14], and [22]). Many combustion studies simply use Fick's law,

$$
\mathbf{J}_{\alpha}=-\rho D_{\alpha} \nabla\left(\rho_{\alpha} / \rho\right)
$$

where $\rho$ is the total density, and $D_{\alpha}$ is the species diffusivity. The original version of COYOTE used this approximation with the same value of $D_{\alpha}$ for all species, and this value was given as the kinematic viscosity of the mixture divided by a constant mixture Schmidt number. This simple model has the advantages that it is easy to program, computationally inexpensive, and the species mass fluxes properly add up to zero when summed over species. Experience suggests that this approximation is adequate for turbulent flows (where molecular transport is overwhelmed by the turbulent eddy diffusion) and in some laminar cases using global chemical kinetics (where the detailed chemical composition is not being modeled all that accurately in any case). In cases where the flow is laminar or nearly so and a detailed chemical reaction network is included, a more accurate model is required.

One such model assumes that multicomponent diffusion in gases is described by the Stefan-Maxwell equations

$$
\sum_{\beta}\left(X_{\alpha} X_{\beta} / D_{\alpha \beta}\right)\left(\mathbf{u}_{\beta}-\mathbf{u}_{\alpha}\right)=\mathbf{G}_{\alpha} \equiv \mathbf{d}_{\alpha}+\chi_{\alpha} \nabla \ln T \quad(\alpha=1, \ldots N),
$$

where $\mathbf{u}_{\alpha}$ is the velocity of species $\alpha$ and $D_{\alpha \beta}$ is the binary diffusivity for the pair of species $(\alpha, \beta)$. The diffusive mass fluxes are given by

$$
\mathbf{J}_{\alpha}=\rho_{\alpha}\left(\mathbf{u}_{\alpha}-\mathbf{u}\right)
$$

where $\mathbf{u}$ is the mass-weighted mixture velocity (calculated by COYOTE's hdyrodynamics package),

$$
\rho \mathbf{u}=\sum_{\alpha} \rho_{\alpha} \mathbf{u}_{\alpha}
$$


The diffusional fluxes could be found by solving the coupled system 12 through 14 for each cell on each time step, a procedure often considered too complex and expensive when more than two species are present. Another approach is to decouple the fluxes from one another with what is called the "effective binary diffusion approximation," which exists in a variety of forms.

Ramshaw [23] has developed an effective binary diffusion approximation that we have implemented in COYOTE as one option. This model simultaneously accounts for all four types of diffusion, and for which the fluxes correctly sum to zero. The resulting fluxes are

$$
\mathbf{J}_{\alpha}=-C M_{\alpha} D_{\alpha} \mathbf{G}_{\alpha}+Y_{\alpha} C \sum_{\beta} M_{\beta} D_{\beta} \mathbf{G}_{\beta},
$$

where $M_{\alpha}$ is the molecular woight of species $\alpha$,

$$
C=\sum_{\alpha} \rho_{\alpha} / M_{\alpha}
$$

is the total molar concentration of the mixture, and

$$
D_{\alpha}=\left(1-X_{\alpha}\right)\left(\sum_{\beta \neq \alpha} X_{\beta} / D_{\alpha \beta}\right)^{-1} .
$$

In practice, we have found direct solution of the Stefan-Maxwell equations to be practical. This is the preferred method of treating mass diffusion in most cases, and it is another option implemented in COYOTE. In this case, the diffusional fluxes are found by solving the coupled linear system 12 through 14 for each cell face on each time step. We use a reference frame moving at velocity $\mathbf{u}$. The basic linear system is equation 12 . The coefficient matrix is singular, so two actions must be taken. First, eliminate all rows of the matrix $X_{\alpha} X_{\beta} / D_{\alpha \beta}$ that are all zeroes. These correspond to species with zcro concentration, and the bookkecping can be dono with a simple link list algorithm. Second, the resulting matrix is still singular, so it is necessary to replace one row of the matrix with equation 14 . We usually sclect the row for the least abundant species. Sometimes there still can be problems with the matrix being ill-conditioned, so in practice it occasionally is necessary also to eliminate rows for species with densities less than some tiny cutoff value, which we normally take to be zero. With very low abundances, it is usually safe to assume the diffusional velocity is zero. In addition, it seems to holp the conditioning to solve for $X_{\alpha} \mathbf{u}_{\alpha}$ rather than for $\mathbf{u}_{\alpha}$, so 
the $\beta$ th row of the coefficient matrix is $X_{\beta} / D_{\alpha \beta}$. Once the linear system is solved for the $\mathbf{u}_{\alpha}$, the fluxes are computed from equation 13 .

The remaining issue is the specification of the binary diffusion coefficients and thermal diffusion coefficients. We follow Hayashi and Hishida [10] in evaluation of the binary diffusion cocfficients:

$$
D_{\alpha \beta}=\frac{3}{16}\left(\frac{2 k_{\mathrm{B}}^{3}}{\pi m_{\mathrm{H}}}\right)^{1 / 2} \frac{\left[T^{3}\left(\frac{M_{\alpha}+M_{\beta}}{M_{\alpha} M_{\beta}}\right)\right]^{1 / 2}}{P \sigma_{\alpha \beta}^{2} \Omega^{(1,1) *}\left(T_{\alpha \beta}^{*}\right)} f_{\mathrm{D}}=1.8829 \times 10^{3} \frac{\left[T^{3}\left(\frac{M_{\alpha}+M_{\beta}}{M_{\alpha} M_{\beta}}\right)\right]^{1 / 2}}{P \sigma_{\alpha \beta}^{2} \Omega^{(1,1) *}\left(T_{\alpha \beta}^{*}\right)},
$$

where $P$ is the total pressure of the mixture and $f_{\mathrm{D}}$ is a correction factor in the range $1.0 \leq f_{\mathrm{D}} \leq 1.1$. We take $f_{\mathrm{D}}=1.0$, and the numerical factor in the right equality assumes cgs units except for $\sigma_{\alpha \beta}$, which is in Ångstroms. We also use

$$
\begin{gathered}
\sigma_{\alpha \beta}=0.5\left(\sigma_{\alpha}+\sigma_{\beta}\right) \\
\Omega^{(1,1) *}\left(T_{\alpha \beta}^{*}\right)=\left(T / T_{e \alpha \beta}\right)^{-0.145}+\left(T / T_{e \alpha \beta}+0.5\right)^{-2}
\end{gathered}
$$

and

$$
T_{e \alpha \beta}=\left(T_{e \alpha} T_{e \beta}\right)^{1 / 2}=\left(\frac{\epsilon_{\alpha} \epsilon_{\beta}}{k_{\mathrm{B}}^{2}}\right)^{1 / 2}
$$

It is common practice to ignore the thermal diffusion torms and set $\chi_{\alpha}=D_{\alpha}^{T}=0$. Not only are thermal diffusion cffects usually small, there are practical difficulties associated with obtaining the required values of $\chi_{\alpha}$. There is a shortage of experimental data, and the traditional theory is so complex as to hinder its application by the nonspecialist. However, Ramshaw has developed an approximate simplified theory [24] that has been incorporated into COYOTE. The first step is to estimate the collision cross section between molecules of types $\alpha$ and $\beta$ as

$$
\sigma^{\alpha \beta}=0.25 \pi\left(\sigma_{\alpha}+\sigma_{\beta}\right)^{2}
$$

We define

$$
\gamma_{\alpha \beta}=\frac{m_{\mathrm{H}}}{2 k_{\mathrm{B}} T} \frac{M_{\alpha} M_{\beta}}{M_{\alpha}+M_{\beta}} .
$$

With these two parameters we estimate the collision time

$$
\tau_{\alpha}=\frac{1}{2}\left[\sum_{\beta=1}^{N} \frac{N_{\beta} \sigma^{\alpha \beta}}{\left(\pi \gamma_{\alpha \beta}\right)^{1 / 2}}\right]^{-1}
$$


where $N_{\beta}$ is the number density of species $\beta$. Next we calculate

$$
\mathcal{B}_{\alpha \beta}=-\begin{gathered}
R T P X_{\alpha} X_{\beta} M_{\alpha} \tau_{\beta} \\
2 D_{\alpha \beta} M_{\beta}\left(M_{\alpha}+M_{\beta}\right)
\end{gathered}
$$

which are in turn used to calculate the $\chi_{\alpha}$ using the left equality of

$$
\chi_{\alpha}=P^{-1} \sum_{\beta=1}^{N}\left(\mathcal{B}_{\beta \alpha}-\mathcal{B}_{\alpha \beta}\right)=\sum_{\beta=1}^{N} \frac{X_{\alpha} X_{\beta}}{D_{\alpha \beta}}\left(\frac{D_{\alpha}^{T}}{\rho_{\alpha}}-\frac{D_{\beta}^{T}}{\rho_{\beta}}\right) .
$$

The $\chi_{\alpha}$ are used in the Stefan-Maxwell equations 12 and 14, which in turn are used with equation 13 to calculate the diffusion fluxes. The second equality in equation 26 is a linear system that can be solved for the $D_{\alpha}^{T}$ for use in evaluating the Dufour term in the encrgy flux. This system is singular, and one equation must be replaced by the constraint

$$
\sum_{\alpha} D_{\alpha}^{T}=0
$$

\section{Transport Coefficients for Plasmas}

The theoretical description discussed above requires a bit of gencralization for the case of plasmas. Evaluation of transport coefficients for a general multicomponent plasma is even more complex and challenging than in the case of neutral molecules. We shall specialize the presentation of Ramshaw and Chang $[25,26]$ and present a model for an electrically noutral, multicomponent, onc-temperature plasma with a zero magnetic field. In a fully or partially ionized plasma, there are nonzero body forces due to an induced clectric field $\mathbf{E}$ that are not present in the un-ionized fluids usually considered in combustion modeling. The clectric field is implicitly determined by the current density $\mathbf{J}_{q}$, which is a linear combination of the diffusional mass fluxes $\mathbf{J}_{\alpha}$. We are concerned with the case $\mathbf{J}_{q}=0$, which is commonly referred to as ambipolar diffusion. Wo shall present a simplified model within the aforementioned constraints that should be adequate as a starting point. Although the current version of COYOTE assumes all species are at the same tempcrature, we shall retain the distinction between ion temperature $T_{i}$ (which we assume is the same for all ions and neutrals) and electron temperature $T_{e}$ in this section. 


\subsection{The Coefficient of Viscosity}

The calculation of the viscosity of a mixture of arbitrarily ionized atoms is not a simple matter. The standard references, such as Mitchner and Kruger [27], p. 413, treat a fully ionized plasma whose atomic charge is $Z_{\alpha}$ in units of the clectron charge $e$. For a single species of ion, this gives a viscosity of

$$
\mu_{\alpha}=\frac{0.406 m_{\alpha}^{1 / 2}\left(k T_{i}\right)^{5 / 2}}{Z_{\alpha}^{4} e^{4} \ln \Lambda}=2.21 \times 10^{-15} \frac{M_{\alpha}^{1 / 2} T_{i}^{5 / 2}}{Z_{\alpha}^{4} \ln \Lambda} \mathrm{g} / \mathrm{cm}-\mathrm{s},
$$

where $m_{i}$ is the ion mass. The cocfficient $2.21 \times 10^{-15}$ is somewhat uncertain. Note that the electron viscosity will normally be much less than a typical ion viscosity. If $N_{e}=\rho_{e} / m_{e}$ is the electron number density, then the electron-ion Coulomb logarithm is [27], pp. 56-58,

$$
\Lambda=\frac{3}{2 Z_{\alpha} e^{3}}\left(\frac{k^{3} T_{e}^{3}}{\pi N_{e}}\right)^{1 / 2} \approx 1.24 \times 10^{4} \frac{T_{e}^{3 / 2}}{Z_{\alpha} N_{e}^{1 / 2}}=1.18 \times 10^{-9} \frac{T_{e}^{3 / 2}}{Z_{\alpha} \rho_{e}^{1 / 2}}
$$

Somov [28] modifies this somewhat to

$$
\begin{gathered}
\Lambda=\frac{3}{2 Z_{\alpha} e^{3}}\left(\frac{k^{3} T_{e}^{3}}{\pi N_{e}}\right)^{1 / 2} \approx 1.24 \times 10^{4} \frac{T_{e}^{3 / 2}}{Z_{\alpha} N_{e}^{1 / 2}} \quad \text { for } T_{e}<5.8 \times 10^{5} \mathrm{~K} \\
=\frac{3}{2 Z_{\alpha} e^{3}}\left(\frac{k^{3} T_{e}^{3}}{\pi N_{e}}\right)^{1 / 2}\left(\frac{5.8 \times 10^{5}}{T_{e}}\right)^{1 / 2} \approx 9.44 \times 10^{6} \frac{T_{e}}{Z_{\alpha} N_{e}^{1 / 2}} \quad \text { for } T_{e} \geq 5.8 \times 10^{5} \mathrm{~K}
\end{gathered}
$$

This expression is valid only if

$$
N_{D}=\frac{4}{3} \pi \lambda_{D}^{3} N_{e} \gg 1
$$

where the electron Debye length is

$$
\lambda_{D}=\left(\frac{k_{B} T_{e}}{4 \pi N_{e} e^{2}}\right)^{1 / 2}=6.9\left(T_{e} / N_{e}\right)^{1 / 2} \mathrm{~cm} .
$$

Lee and More [29] suggest setting a minimum value of 2.0 on the Coulomb logarithm when calculating conductivities with their model (to be described in the next subsection), and we adopt that suggestion here as well.

For other conditions, it will be necessary to use other approximations for the Coulomb logarithm, or perhaps a different formalism altogether. For example, the case of dense, degenerate plasmas found in the interiors of white dwarf stars is treated by Itoh, Kohyama, and Takeuchi [30]. These extreme conditions are beyond the scope of this report. 
Of course, prediction of the mixture viscosity for the partially ionized case is an even more difficult problem than for a single ionic species. Mitchner and Kruger provide a theoretical discussion that fails to provide a computationally convenient formalism. So, we use a very approximate, somewhat ad hoc model. First, we assume the species viscosities for the neutrals are all given by equation 2 or 3 . Then we calculate a species viscosity for each charged species using equations 28 and 30 , assuming $T_{i}=T_{e}=T$. The same value of $\Lambda$ will be used for all transport coefficients in a given cell, using a number-weighted averaged charge $Z^{*}$ in place of $Z_{\alpha}$. If $\alpha^{\prime}$ represents the set of species indices for all charged species except electrons, then we define

$$
Z^{*}=\frac{\sum_{\alpha^{\prime}}\left|Z_{\alpha^{\prime}}\right| N_{\alpha^{\prime}}}{\sum_{\alpha^{\prime}} N_{\alpha^{\prime}}} \equiv \frac{1}{N_{i}} \sum_{\alpha^{\prime}}\left|Z_{\alpha^{\prime}}\right| N_{\alpha^{\prime}}
$$

Then the mixture rule given by equations 5 and 6 is used to compute the mixture viscosity.

When the radiation energy density is large, as it is in stellar interiors, there are additional considerations. In the gray one-temperature approximation, the viscous stresses must include a contribution from the radiation $[31,32]$. This model requires the radiative viscosity

$$
\mu_{\mathrm{r}}=4 a T^{4} / 15 c \kappa \rho
$$

where $\kappa$ is the Rosseland mean opacity, $a=7.563 \times 10^{-15} \mathrm{crgs} /\left(\mathrm{cm}^{3} \mathrm{~K}^{4}\right)$ is the StefanBoltzmann constant, and $c=2.998 \times 10^{10} \mathrm{~cm} / \mathrm{s}$ is the speed of light. Numerous opacity tables are found in the astrophysics literature, for example $[33,34,35]$. It is beyond the scope of this report to attempt to provide any kind of critical summary of this literature. Also, we shall make no attempt to discuss more detailed models of radiation effects.

\subsection{Thermal Conductivities}

Mitchner and Kruger [27] and Chapman and Cowling [22] (p. 179) give the thermal conductivity as

$$
K_{\alpha}=\frac{5}{2} \mu_{\alpha} C_{v \alpha}
$$

This is the same as the Eucken correction, equation 9 , with $\gamma_{\alpha}=5 / 3$. We use this approximation for the ions. 
In general, when energy is transported via conduction in a plasma with $T>2 \times 10^{4} \mathrm{~K}$, electron conduction plays a greater role than that of the ions due to their greater mobility. To calculate the conduction coefficients, a perturbation solution to a Boltzmann transport equation with a linearized collision operator is found [36, 37]. For the clectron conductivity, electron-electron collisions are generally not explicitly included in this operator since electronion collisions are usually more efficient in spatially transporting electron energy because of a higher average momentum transfer. An expression for the electron conductivity derived in this fashion incorporating degeneracy as well as partial ionization effects is given by Lee and More [29] as

$$
K_{e}=\frac{3 k_{B}\left(k_{B} T_{e}\right)^{5 / 2} N_{e}}{2^{3 / 2} m_{e}^{1 / 2} \pi Z^{* 2} N_{i} e^{4} \ln \Lambda} A^{\beta}\left(\mu / k_{B} T_{e}\right)\left[1+\exp \left(-\mu / k_{B} T_{e}\right)\right] F_{1 / 2}\left(\mu / k_{B} T_{e}\right),
$$

where $\ln \Lambda \geq 2$. The coefficients involving the chemical potential $\mu$ may be fit in terms of the degeneracy parameter $y=\ln \left[1+\exp \left(\mu / k_{B} T_{e}\right)\right]$ by

$$
A^{\beta}\left(\mu / k_{B} T_{e}\right)=\frac{13.581+0.976 y+0.437 y^{2}}{1.0+0.510 y+0.126 y^{2}} .
$$

There are two ways of computing the degeneracy parameter and the Fermi-Dirac integral. First, the Fcrmi-Dirac integral may be approximated in terms of $y$ by

$$
\left[1+\exp \left(-\mu / k_{B} T_{e}\right)\right] F_{1 / 2}\left(\mu / k_{B} T_{e}\right)=\frac{\pi^{1 / 2}}{2}+0.2 y^{1 / 2}-0.16 y+\frac{2 y^{3 / 2}}{3} .
$$

The constant term on the right hand side makes the fit exact as $y$ approaches zero (nondegenerate), and the last term makes the fit exact at $y$ goes to infinity (complete nonrelativistic degeneracy). Comparing with accurate results for partial degeneracy [38], we find this fit has a maximum error of approximately 3 percent around $\mu / k_{B} T_{e}=0$. The degeneracy factor is fit by

$$
y=\frac{x^{3}\left(0.753+0.168 x+0.310 x^{2}\right)}{1.0+0.270 x+0.228 x^{2}+0.310 x^{3}},
$$

where $x=\left(E_{f} / k_{B} T_{e}\right)^{1 / 2}$, and

$$
E_{f}=\frac{\hbar^{2}}{2 m_{e}}\left(3 \pi^{2} N_{e}\right)^{2 / 3}
$$

is the electron Fermi energy.

I prefer a different procedure for handling the degeneracy parameter and Fermi-Dirac integrals that avoids the marginally adequate fits given by equations 38-40. The CFD code 
inputs to the transport cocfficient routine the electron temperature $T_{e}$ and the electron number density $N_{e}$. The nonrelativistic degenerate electron equation of state [39] then gives $F_{1 / 2}(\eta)$, where $\eta=\mu / k_{B} T_{e}$, from

$$
N_{e}=\frac{4 \pi}{h^{3}}\left(2 m_{e} k_{B} T_{e}\right)^{3 / 2} F_{1 / 2}(\eta)
$$

Then we can use highly accurate tables [38] or functional fits [40] to invert $F_{1 / 2}(\eta)$ to find $\eta$. The latter are especially convenient. Then we trivially compute $y$ and $A^{\beta}(\eta)$ from equation 37 and finally the conductivity from equation 36 .

Hubbard and Lampe [41] is the standard reference for clectron thermal conduction opacities in stellar interiors. For extreme conditions, such as found in the interiors of white dwarf stars, this is the recommended thermal conductivity. Canuto [42] extends this work to include relativistic effects.

In situations with high radiation encrgy density, the radiative heat flux must be included $[31,32]$. In the gray one-temperature approximation, the radiative flux is

$$
\mathrm{q}_{\mathrm{r}}=-K_{\mathrm{r}} \nabla T=-\frac{4 a c T^{3}}{3 \kappa \rho} \nabla T
$$

Once again, further consideration of radiative effects is beyond the scope of this report.

\subsection{Binary Diffusion Coefficients}

For mass diffusion in plasmas, the Stefan-Maxwell equations must be generalized to include a body force term representing the elcctric field set up by ambipolar diffusion:

$$
\mathbf{d}_{\alpha}=\nabla X_{\alpha}+\left(X_{\alpha}-Y_{\alpha}\right) \frac{\nabla P}{P}-\frac{1}{P} \rho_{\alpha} q_{\alpha} \mathrm{E}
$$

where $q_{\alpha}=Z_{\alpha} e A / M_{\alpha}$ is the charge per unit mass of species $\alpha$. Here $A=6.022 \times 10^{23}$ is Avogadro's number. Here we have made use of the charge neutrality condition $\sum_{\alpha} \rho_{\alpha} q_{\alpha}=0 .{ }^{1}$ The Stefan-Maxwell equations 12 remain unchanged except for the new $\mathbf{d}_{\alpha}$ defined by cquation 43. The coefficient matrix, howcver, picks up one new column for the coefficients of the unknown electric field $\mathbf{E}$. The assumption of zero magnetic field implies that the current $\mathbf{J}_{q}=\sum_{\alpha} \rho_{\alpha} q_{\alpha} \mathbf{u}_{\alpha}=0$ by Ampere's Law. The lincar system now must include the zero-current

\footnotetext{
${ }^{1}$ In some problems (for example, [43]), it may be necessary to include an additional radiative force as part of $\mathbf{F}_{c}$, but we shall not consider that possibility any further in this report.
} 
constraint as the additional equation which allows us to solve for the electric field $\mathrm{E}$ along with the diffusion velocities.

To complete the mass transport model, we need binary diffusion coefficients and thermal diffusion coefficients. For pairs of neutral molecules, we continue using equations 18 through 27. For a charged-neutral pair, we also use the neutral-species formalism for the binary diffusion coefficient. In all other cases, we use the model of Ramshaw $[44,45]$, which has been modified to accomodate tho Coulomb potential.

Charged pairs are not described accurately by first-order Chapman-Enskog theory, and a correction factor $f_{\alpha \beta}$ must be applied to obtain

$$
D_{\alpha \beta}=\frac{3 f_{\alpha \beta} k_{B}^{2} T_{\alpha} T_{\beta}}{16 P \mu_{\alpha \beta} \Omega_{\alpha \beta}^{(1,1)}\left(T_{\alpha \beta}\right)}
$$

where $\mu_{\alpha \beta}=m_{\alpha} m_{\beta} /\left(m_{\alpha}+m_{\beta}\right)$ is the reduced mass. The factor $f_{\alpha \beta}=1$ if $\alpha, \beta \neq e$, and $f_{\alpha e}=f_{e \alpha}=f\left(Z_{\alpha}\right)$, where $f(1)=1.97, f(2)=2.33$, and $f(3)=2.53$. The collision integral is

$$
\Omega_{\alpha \beta}^{(1,1)}\left(T_{\alpha \beta}\right)=\left(\frac{\pi}{\mu_{\alpha \beta}}\right)^{1 / 2} \frac{Z_{\alpha} Z_{\beta} e^{2}}{\left(2 k_{B} T_{\alpha \beta}\right)^{3 / 2}} \ln \Lambda .
$$

The thermal diffusion coefficient for charged pairs is defined in terms of the inverse of the collision frequency,

$$
\tau_{\alpha}=0.5\left[2 \delta_{\alpha e} N_{e} \sigma_{e e}\left(\frac{k_{B} T_{e}}{\pi m_{e}}\right)^{1 / 2}+\sum_{\beta \neq e} \frac{N_{\beta} \sigma_{\alpha \beta}}{\left(\pi \gamma_{\alpha \beta}\right)^{1 / 2}}\right]^{-1}
$$

where

$$
\begin{gathered}
\sigma_{\alpha \beta}=\frac{2 \pi}{3}\left(\frac{Z_{\alpha} Z_{\beta} e^{2}}{k_{B} T_{\alpha \beta}}\right)^{2} \ln \Lambda, \\
\gamma_{\alpha}=m_{\alpha} / 2 k_{B} T_{\alpha},
\end{gathered}
$$

$\gamma_{\alpha \beta}$ is defined by equation 23 , and $\delta_{e \alpha}$ is the Kronecker delta. Then

$$
\mathcal{B}_{\alpha \beta}=\frac{3 f_{\alpha \beta} P_{\alpha} P_{\beta} \tau_{\beta} \gamma_{\alpha \beta}}{4 P \gamma_{\beta}^{2} D_{\alpha \beta}}
$$

which differs from the neutral-neutral case by a factor of $-3 f_{\alpha \beta}$.

Ramshaw [45] argues that a charged-neutral pair has a $r_{\alpha \beta}^{-5}$ potential except at very close range, so the thermal diffusion coefficients should be very small or zero. Therefore, we set the thermal diffusion coefficient $\mathcal{B}_{\alpha \beta}=0$ if $\alpha, \beta$ are a charged-neutral pair. 
A necessary condition for this formalism to be valid is the ideal gas limit. For more extreme conditions, a more complex formalism is necessary. A lot of work has been done for the conditions found in stellar interiors. Aller and Chapman [46] present a simple cxample including ambipolar diffusion in the sun. A rather arbitrary selection of more complex formalisms, including radiative forces, is given in [43] and [47]-[52].

\section{Concluding Comments}

This report presents a homogeneous compilation of transport coefficients from disparate sources. The compilation originally was oriented mainly toward combustion applications, but the present version extends the database to include plasmas under conditions appropriate to plasma torches and certain astrophysical phenomena. However, the information should be applicable to any collision-dominated fluid or plasma within the ideal gas regime. The model has been implemented in COYOTE, and simuluations with up to 27 species have been completed without difficulty or excessive computational expense.

A natural question is how well the collected formulas and tables represent reality. Unfortunately, it is not casy to answer this question. For some gases, the problem is the shear volume of data found in the literature. For some of these, there are publications devoted to a critical evaluation of the literature and presentation of correlations and tables of recommended values. Often these are accurate to within a few percent over a wide range of conditions. Even in those cases, however, there can still be some discrepancies in the reported Lennard-Jones parameters. This can be seen in Table 3 where there are several examples of multiple entries for a given species, each entry from a different source. For example, there are two entries for $\mathrm{H}_{2}$. Transport coefficients depend only weakly on $\epsilon / k_{\mathrm{B}}$, so both entries produce comparable results. Although the Lennard-Jones model is reasonably accurate (the viscosity is approximately 6 percent high at $320 \mathrm{~K}$, depending on which experimental value one chooses), the generalized Sutherland formula (Table 1) is slightly more accurate. Even

in the case of $\mathrm{H}$, the two sets of Lennard-Jones parameters produce transport coefficients that are not as different as one might expect. The first set appears to be somewhat more accurate for binary diffusion coefficients, but the second set is definitely superior for viscosity and thermal conductivity. Caveat emptor. 
At the other extreme, there is a scarcity of experimental data for unstable or highly reactive species such as $\mathrm{OH}$. Fortunately, these species are not sufficiently abundant in most combustion systems that they significantly impact the viscosity or thermal conductivity. However, some of the binary diffusion coefficients, such as those involving $H$, have important effects on flame propagation speeds and structure. Appropriate experimental data for plasmas appear to be very scarce, and the theoretical estimates presented here have received minimal testing.

There is one final comment about the use of the Lennard-Jones model. CHEMKIN, a widcly used combustion code, has a large database of Lennard-Jones parameters. However, the first step in running a problem is to have the code convert the Lennard-Jones transport coefficients into polynomial fits. We prefer the direct use of the model equations since this procedure avoids the introduction of some unnecessary truncation errors. However, it may be necessary to reconsider this choice if we ever need to simulate extreme conditions, such as the interior of a white dwarf star, where a different formalism is appropriate.

\section{Acknowledgements}

I thank John Ramshaw for many useful discussions on various aspects of including molecular transport effects into computational fluid dynamics programs. I thank C. H. Chang for sharing his experience with plasma transport coefficients and Nick Marinov for help with CHEMKIN. Thanks also to Shaheen Tonse for discussions on the accuracy of the LennardJones model. This work was performed under the auspices of the U. S. Department of Energy by University of California Lawrence Livermore National Laboratory under contract No. W-7405-Eng-48.

\section{References}

[1] J. O. Hirschfelder, C. F. Curtiss, and R. B. Bird, Molecular Theory of Gases and Liquids (Wilcy, New York, 1964).

[2] T. P. Coffee and J. M. Heimerl, "Transport algorithms for premixed, laminar steadystate flames," Comb. \& Flame 43, 273 (1981). 
[3] G. Dixon-Lewis, "Flame structure and flame reaction kineitcs II. Transport phenomena in multicomponent systems," Proc. Roy. Soc. A 307, 111 (1968).

[4] R. J. Kee, J. A. Miller, and T. H. Jefferson, "CHEMKIN: A general-purpose, problemindependent, transportable, Fortran chemical kinetics code package," Sandia National Laboratories Report No. SAND80-8003, 1980.

[5] R. J. Kee, G. Dixon-Lewis, J. Warnatz, M. E. Coltrin, and J. A. Miller, "A Fortran computer code package for the evaluation of gas-phase multicomponent transport properties," Sandia National Laboratories Report No. SAND86-8246, 1986; reprinted 1990 and 1995.

[6] R. J. Kee, F. M. Rupley, J. A. Miller, "The Chemkin thermodynamic data base," Sandia National Laboratories Report No. SAND87-8215, 1987.

[7] R. J. Kee, F. M. Rupley, J. A. Miller, "Chemkin-II: A Fortran chemical kinetics package for the analysis of gas-phase chemical kinetics," Sandia National Laboratories Report No. SAND89-8009, 1989; reprinted 1990.

[8] P. H. Paul, "DRFM: A new package for the evaluation of gas-phase-transport propertics," Sandia National Laboratories report SAND98-8203, 1997.

[9] L. D. Cloutman, "COYOTE: A computer program for 2D reactive flow simulations," Lawrence Livermore National Laboratory Report No. UCRL-ID-103611, 1990.

[10] A. K. Hayashi and M. Hishida, "Numerical study on pulsed jet combustion," in Fourth International Symposium on Computational Fluid Dynamics, Vol. III, University of California at Davis, Sept. 9-12, 1991, pp. 19-24.

[11] F. M. White, Viscous Fluid Flow (McGraw-Hill, New York, 1974).

[12] L. Monchick and E. A. Mason, "Transport properties of polar gases," J. Chem. Phys. $35,1676(1961)$.

[13] C. R. Wilke, "A viscosity equation for gas mixtures," J. Chem. Phys. 18, 517 (1950). 
[14] R. B. Bird, W. E. Stewart, and E. N. Lightfoot, Transport Phenomena (Wilcy, New York, 1960).

[15] W. Behrendt, N. Baumann, H.-J. Fachmann, P. Kuhn, and P. Mcrlet, Gmelin Handbuch der Anorganischen Chemie, 8th cd., edited by H. Bitterer (Springer-Verlag, Berlin, 1978), Suppl. Vol. 2, pp. 143-146.

[16] P. A. Thompson, "Compressible-Fluid Dynamics" (New York: McGraw-Hill), 1972.

[17] R. C. Weast, Ed., CRC Handbook of Chemistry and Physics, 60th Edition (CRC Press, Boca Raton, 1979).

[18] E. Oran, T. Young, and J. Boris, "Application of time-dependent numcrical methods to the description of reactive shocks," in Proceedings of the 17th Symposium (International) on Combustion (The Combustion Institute, Pittsburgh, 1979), pp. 43-54.

[19] J. H. Ferziger and H. G. Kaper, Mathematical Theory of Transport Processes in Gases (North-Holland, Amsterdam, 1972).

[20] S. Mathur, P. K. Tondon, and S. C. Saxena, "Thermal conductivity of binary, ternary, and quaternary mixtures of rare gases," Mol. Phys. 12, 569 (1967).

[21] R. S. Brokaw, "Approximate formulas for the viscosity and thermal conductivity of gas mixturcs," J. Chem. Phys. 29, 391 (1958).

[22] S. Chapman and T. G. Cowling, The Mathematical Theory of Non-Uniform Gases (Cambridge University Press, London, 1952).

[23] J. D. Ramshaw, "Self-consistent effective binary diffusion in multicomponent gas mixtures," J. Non-Equilib. Thermodyn. 15, 295 (1990).

[24] J. D. Ramshaw, "Hydrodynamic theory of multicomponent diffusion and thermal diffusion in multitemperature gas mixtures," J. Non-Equilib. Thermodyn. 18, 121 (1993).

[25] J. D. Ramshaw and C. H. Chang, "Ambipolar diffusion in multicomponent plasmas," Plasma Chem. Plasma Process. 11, 395 (1991). 
[26] J. D. Ramshaw and C. H. Chang, "Multicomponent diffusion in two-tempcrature magnetohydrodynamics," Phys. Rev. E 53, 6382 (1996).

[27] M. Mitchner and C. H. Kruger, Jr., Partially Ionized Gases (Wiley-Interscience: New York, 1973).

[28] B. V. Somov, Physical Processes in Solar Flares, (Kluwer, Dordrecht, 1992).

[29] Y. T. Lee and R. M. More, "An electron conductivity model for dense plasmas," Phys. Fluids 27, 1273 (1984).

[30] N. Itoh, Y. Kohyama, and H. Takeuchi, "Viscosity of dense matter," Ap. J. 317, 733 (1987).

[31] P. Ledoux and Th. Walraven, "Variable stars," in: Handbuch der Physik, Volume 51, (ed.) S. Flügge (Springer-Verlag, Berlin, 1958), p. 353. See Section C, p. 431ff.

[32] J.-L. Tassoul, "Theory of Rotating Stars" (Princeton, NJ: Princeton U. Press), 1978.

[33] A. N. Cox and J. N. Stewart, "Rosseland opacity tables for Population II compositions," Ap. J. Suppl. 19, No. 174, 261 (1970).

[34] A. N. Cox and J. E. Tabor, "Radiative opacity tables for 40 stellar mixtures," Ap. J. Suppl. 31, 271 (1976).

[35] F. J. Rogers and C. A. Iglesias, "Radiative atomic Rosseland mean opacity tables," Ap. J. Suppl. 79, 507 (1992).

[36] S. I. Braginskii, "Transport phenomena in a completcly ionized two-temperature plasma, Sov. Phys. JETP 6, 358 (1958).

[37] S. I. Braginskii, "Transport processes in a plasma," in Reviews of Plasma Physics, edited by M. A. Leontovich (Consultants Bureau, New York, 1965), p. 205.

[38] L. D. Cloutman, "Numerical evaluation of the Fermi-Dirac integrals," Ap. J. Suppl. 71, 677 (1989). 
[39] S. Chandrasekhar, Stellar Structure (University of Chicago Press, Chicago, 1939).

[40] H. M. Antia, "Rational function approximations for Fermi-Dirac integrals," Ap. J. Suppl. 84, 101 (1993).

[41] W. B. Hubbard and M. Lampe, "Thermal conduction by electrons in stellar matter," Ap. J. Suppl. 18, No. 163, 297 (1969).

[42] V. Canuto, "Electrical conductivity and conductive opacity of a relativistic electron gas," Ap. J. 159, 641 (1970).

[43] G. Michaud, "Diffusion processes in peculiar A stars," Ap. J. 160, 641 (1970).

[44] J. D. Ramshaw, "Simple approximation for thermal diffusion in gas mixtures," J. NonEquilib. Thermodyn. 21, 99 (1996).

[45] J. D. Ramshaw, "Simple approximation for thermal diffusion in ionized gas mixtures," J. Non-Equilib. Thermodyn. 21, 233 (1996).

[46] L. H. Aller and S. Chapman, "Diffusion in the sun," Ap. J. 132, 461 (1960).

[47] G. Michaud, Y. Charland, S. Vauclair, and G. Vauclair, "Diffusion in main-scquence stars: Radiation forces, time scales, anomalies," Ap. J. 210, 447 (1976).

[48] P. D. Noerdlinger, "Automatic computation of diffusion rates," Ap. J. Suppl. 36, 259 (19978).

[49] R. Roussel-Dupré, "Computations of ion diffusion coefficients from the BoltzmannFokker-Planck equation," Ap. J. 243, 329 (1981).

[50] D. Muchmore, "Diffusion in white dwarf stars," Ap. J. 278, 769 (1984).

[51] C. Paquette, C. Pelletier, G. Fontaine, and G. Michaud, "Diffusion coefficients for stellar plasmas," Ap. J. Suppl. 61, 177 (1986).

[52] A. A. Thoul, J. N. Bahcall, and A. Locb, "Element diffusion in the solar interior," Ap. J. 421, 828 (1994). 


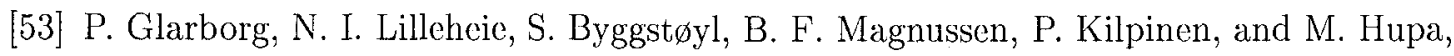
"A reduced mechanism for nitrogen chemistry in methane combustion," in Proceedings of the 24th Symposium (International) on Combustion (The Combustion Institute, Pittsburgh, 1993), in press.

[54] J. Bzowski, J. Kestin, E. A. Mason, and F. J. Uribe, "Equilibrium and transport properties of gas mixtures at low density: eleven polyatomic gases and five noble gases," J. Phys. Chom. Ref. Data 19, 1179 (1990).

[55] A. M. Dean, J. W. Bozclli, and E. R. Ritter, "CHEMACT: A computer code to estimate rate constants for chemically-activated reactions," Combust. Sci. and Tech. 80 , 63 (1991).

[56] R. B. Bird, J. O. Hirschfelder, and C. F. Curtiss, "Theoretical calculation of the equation of state and transport properties of gases and liquids," Trans. ASME 76, 1011 (1954). 
Table 3. Lennard-Jones Parameters for Selected Species

\begin{tabular}{|c|c|c|c|c|c|c|}
\hline Species & Index & $\epsilon / k_{\mathrm{B}}(\mathrm{K})$ & $\sigma(\ddot{A})$ & $\mu$ (Debye) & $\alpha\left(\AA^{3}\right)$ & $Z_{\text {rot }}(298 K)$ \\
\hline $\mathrm{He}$ & 0 & 10.200 & 2.576 & 0.000 & 0.000 & 0.000 \\
\hline $\mathrm{He}$ & 0 & 10.400 & 2.610 & 0.000 & 0.200 & - \\
\hline $\mathrm{Ne}$ & 0 & 35.60 & 2.749 & 0.000 & 0.000 & 0.000 \\
\hline $\mathrm{Ne}$ & 0 & 42.00 & 2.755 & 0.000 & 0.400 & - \\
\hline $\mathrm{Ar}$ & 0 & 136.500 & 3.330 & 0.000 & 0.000 & 0.000 \\
\hline $\mathrm{Ar}$ & 0 & 119.8 & 3.405 & 0.000 & 0.000 & 0.000 \\
\hline $\mathrm{Ar}$ & 0 & 143.200 & 3.350 & 0.000 & 1.642 & - \\
\hline $\mathrm{Kr}$ & 0 & 176.000 & 3.679 & - & - & 0.000 \\
\hline $\mathrm{Kr}$ & 0 & 197.800 & 3.571 & 0.000 & 2.490 & - \\
\hline $\mathrm{Xe}$ & 0 & 229.800 & 3.937 & - & - & 0.000 \\
\hline $\mathrm{Xe}$ & 0 & 274.000 & 3.885 & 0.000 & 4.040 & - \\
\hline $\mathrm{C}$ & 0 & 71.400 & 3.298 & 0.000 & 0.000 & 0.000 \\
\hline $\mathrm{C}_{2}$ & 1 & 97.530 & 3.621 & 0.000 & 1.760 & 4.000 \\
\hline $\mathrm{CH}$ & 1 & 80.000 & 2.750 & 0.000 & 0.000 & 1.000 \\
\hline $\mathrm{CH}_{2}$ & 1 & 144.000 & 3.800 & 0.000 & 0.000 & 1.000 \\
\hline${ }^{1} \mathrm{CH}_{2}$ & 1 & 144.000 & 3.800 & 0.000 & 0.000 & 0.000 \\
\hline $\mathrm{CH}_{3}$ & 1 & 144.000 & 3.800 & 0.000 & 0.000 & 1.000 \\
\hline $\mathrm{CH}_{4}$ & 2 & 141.400 & 3.746 & 0.000 & 2.600 & 13.000 \\
\hline $\mathrm{CH}_{4}$ & 2 & 161.400 & 3.721 & 0.000 & 2.600 & - \\
\hline $\mathrm{CH}_{3} \mathrm{OH}$ & 2 & 481.800 & 3.626 & 0.000 & 0.000 & 1.000 \\
\hline $\mathrm{CH}_{2} \mathrm{O}$ & 2 & 498.000 & 3.590 & 0.000 & 0.000 & 2.000 \\
\hline $\mathrm{CH}_{2} \mathrm{OH}$ & 2 & 417.000 & 3.690 & 1.700 & 0.000 & 2.000 \\
\hline $\mathrm{C}_{2} \mathrm{H}_{2}$ & 1 & 209.000 & 4.100 & 0.000 & 0.000 & 2.500 \\
\hline $\mathrm{C}_{2} \mathrm{H}_{2}$ & 1 & 265.300 & 3.721 & 0.000 & 0.000 & 2.500 \\
\hline $\mathrm{C}_{2} \mathrm{H}_{4}$ & 2 & 280.800 & 3.971 & 0.000 & 0.000 & 1.500 \\
\hline $\mathrm{C}_{2} \mathrm{H}_{4}$ & 2 & 238.400 & 3.496 & 0.000 & 0.000 & 1.500 \\
\hline $\mathrm{C}_{2} \mathrm{H}_{6}$ & 2 & 252.300 & 4.302 & 0.000 & 0.000 & 1.500 \\
\hline $\mathrm{C}_{3} \mathrm{H}_{8}$ & 2 & 266.800 & 4.982 & 0.000 & 0.000 & 1.000 \\
\hline $\mathrm{CN}$ & 1 & 75.000 & 3.856 & 0.000 & 0.000 & 1.000 \\
\hline $\mathrm{CO}$ & 1 & 98.100 & 3.650 & 0.000 & 1.950 & 1.800 \\
\hline $\mathrm{CO}$ & 1 & 98.400 & 3.652 & 0.1098 & 1.950 & - \\
\hline $\mathrm{CO}_{2}$ & 1 & 244.000 & 3.763 & 0.000 & 2.650 & 2.100 \\
\hline $\mathrm{CO}_{2}$ & 1 & 245.300 & 3.769 & 0.000 & 2.650 & - \\
\hline $\mathrm{CF}_{4}$ & 2 & 156.500 & 4.579 & - & - & - \\
\hline $\mathrm{H}$ & 0 & 145.000 & 2.050 & 0.000 & 0.000 & 0.000 \\
\hline $\mathrm{H}$ & 0 & 5.420 & 3.288 & 0.000 & 0.667 & - \\
\hline $\mathrm{H}_{2}$ & 1 & 38.000 & 2.920 & 0.000 & 0.790 & 280.000 \\
\hline $\mathrm{H}_{2}$ & 1 & 23.960 & 3.063 & 0.000 & 0.803 & - \\
\hline $\mathrm{HCN}$ & 1 & 569.000 & 3.630 & 0.000 & 0.000 & 1.000 \\
\hline $\mathrm{HCO}$ & 2 & 498.000 & 3.590 & 0.000 & 0.000 & 1.000 \\
\hline $\mathrm{H}_{2} \mathrm{O}$ & 2 & 572.400 & 2.605 & 1.844 & 0.000 & 4.000 \\
\hline
\end{tabular}


Table 3. Continued.

\begin{tabular}{|c|c|c|c|c|c|c|c|}
\hline Species & Index & $\epsilon / k_{\mathrm{B}}(\mathrm{K})$ & $\sigma(\AA)$ & $\mu$ (Debyc) & $\alpha\left(\AA^{3}\right)$ & $Z_{\text {rot }}(298 K)$ & Ref. \\
\hline $\mathrm{H}_{2} \mathrm{O}$ & 2 & 535.210 & 2.673 & 1.847 & 1.450 & - & {$[8]$} \\
\hline $\mathrm{H}_{2} \mathrm{O}_{2}$ & 2 & 107.400 & 3.458 & 0.000 & 0.000 & 3.800 & \\
\hline $\mathrm{H}_{2} \mathrm{O}_{2}$ & 2 & 368.110 & 3.499 & 1.573 & 2.230 & - & {$[8]$} \\
\hline $\mathrm{HO}_{2}$ & 2 & 107.400 & 3.458 & 0.000 & 0.000 & 1.000 & \\
\hline $\mathrm{HO}_{2}$ & 2 & 365.560 & 3.433 & 2.090 & 1.950 & - & $3]$ \\
\hline $\mathrm{N}$ & 0 & 71.400 & 3.298 & 0.000 & 0.000 & 0.000 & \\
\hline N & 0 & 74.500 & 3.360 & 0.000 & 1.110 & $=$ & 8] \\
\hline $\mathrm{N}_{2}$ & 1 & 97.530 & 3.621 & 0.000 & 1.760 & 4.000 & \\
\hline $\mathrm{N}_{2}$ & 1 & 98.400 & 3.652 & 0.000 & 1.750 & - & 8 \\
\hline $\mathrm{NCO}$ & 1 & 232.400 & 3.828 & 0.000 & 0.000 & 1.000 & \\
\hline NH & 1 & 80.000 & 2.650 & 0.000 & 0.000 & 4.000 & \\
\hline $\mathrm{NH}_{2}$ & 2 & 80.000 & 2.650 & 0.000 & 2.260 & 4.000 & \\
\hline $\mathrm{NH}_{3}$ & 2 & 481.000 & 2.920 & 1.470 & 0.000 & 10.000 & \\
\hline $\mathrm{N}_{2} \mathrm{O}$ & 1 & 232.400 & 3.828 & 0.000 & 0.000 & 1.000 & \\
\hline $\mathrm{N}_{2} \mathrm{O}$ & 1 & 266.800 & 3.703 & 0.1687 & 3.000 & - & 3] \\
\hline NO & 1 & 97.530 & 3.621 & 0.000 & 1.760 & 4.000 & \\
\hline NO & 1 & 125.000 & 3.474 & 0.1578 & 1.740 & - & \\
\hline $\mathrm{NO}_{2}$ & 2 & 200.000 & 3.500 & 0.000 & 0.000 & 1.000 & \\
\hline $\mathrm{NO}_{2}$ & 2 & 204.880 & 3.922 & 0.320 & 3.000 & - & 8) \\
\hline $\mathrm{O}$ & 0 & 80.000 & 2.750 & 0.000 & 0.000 & 0.000 & \\
\hline $\mathrm{O}$ & 0 & 57.910 & 3.064 & 0.000 & 0.802 & - & $3]$ \\
\hline $\mathrm{O}_{2}$ & 1 & 107.400 & 3.458 & 0.000 & 1.600 & 3.800 & \\
\hline $\mathrm{O}_{2}$ & 1 & 121.100 & 3.470 & 0.000 & 1.600 & - & $8]$ \\
\hline $\mathrm{OH}$ & 1 & 80.000 & 2.750 & 0.000 & 0.000 & 0.000 & \\
\hline $\mathrm{OH}$ & 1 & 281.270 & 3.111 & 1.655 & 0.980 & - & 3] \\
\hline $\mathrm{C}_{2} \mathrm{O}$ & 1 & 232.400 & 3.828 & 0.000 & 0.000 & 1.000 & \\
\hline $\mathrm{CN}_{2}$ & 1 & 232.400 & 3.828 & 0.000 & 0.000 & 1.000 & \\
\hline $\mathrm{C}_{2} \mathrm{H}$ & 1 & 209.000 & 4.100 & 0.000 & 0.000 & 2.500 & \\
\hline $\mathrm{C}_{2} \mathrm{H}_{2} \mathrm{OH}$ & 2 & 224.700 & 4.162 & 0.000 & 0.000 & 1.000 & \\
\hline $\mathrm{C}_{2} \mathrm{H}_{3}$ & 2 & 209.000 & 4.100 & 0.000 & 0.000 & 1.000 & \\
\hline $\mathrm{C}_{2} \mathrm{H}_{4}$ & 2 & 280.800 & 3.971 & 0.000 & 0.000 & 1.500 & \\
\hline $\mathrm{C}_{2} \mathrm{H}_{5}$ & 2 & 252.300 & 4.302 & 0.000 & 0.000 & 1.500 & \\
\hline $\mathrm{C}_{2} \mathrm{~N}$ & 1 & 232.400 & 3.828 & 0.000 & 0.000 & 1.000 & \\
\hline $\mathrm{C}_{2} \mathrm{~N}_{2}$ & 1 & 349.000 & 4.361 & 0.000 & 0.000 & 1.000 & \\
\hline $\mathrm{C}_{3} \mathrm{H}_{2}$ & 2 & 209.000 & 4.100 & 0.000 & 0.000 & 1.000 & \\
\hline $\mathrm{C}_{3} \mathrm{H}_{4}$ & 1 & 252.000 & 4.760 & 0.000 & 0.000 & 1.000 & \\
\hline$p-\mathrm{C}_{3} \mathrm{H}_{4}$ & 1 & 252.000 & 4.760 & 0.000 & 0.000 & 1.000 & \\
\hline $\mathrm{C}_{3} \mathrm{H}_{6}$ & 2 & 266.800 & 4.982 & 0.000 & 0.000 & 1.000 & \\
\hline $\mathrm{C}_{3} \mathrm{H}_{7}$ & 2 & 266.800 & 4.982 & 0.000 & 0.000 & 1.000 & \\
\hline $\mathrm{C}_{4} \mathrm{H}_{6}$ & 2 & 357.000 & 5.180 & 0.000 & 0.000 & 1.000 & \\
\hline$i-\mathrm{C}_{3} \mathrm{H}_{7}$ & 2 & 266.800 & 4.982 & 0.000 & 0.000 & 1.000 & \\
\hline
\end{tabular}


Table 3. Continued.

\begin{tabular}{|c|c|c|c|c|c|c|}
\hline Species & Index & $\epsilon / k_{\mathrm{B}}(\mathrm{K})$ & $\sigma(\AA)$ & $\mu$ (Debye) & $\alpha\left(\AA^{3}\right)$ & $Z_{\text {rot }}(298 K)$ \\
\hline$n-\mathrm{C}_{3} \mathrm{H}_{7}$ & 2 & 266.800 & 4.982 & 0.000 & 0.000 & 1.000 \\
\hline $\mathrm{C}_{4} \mathrm{H}$ & 1 & 357.000 & 5.180 & 0.000 & 0.000 & 1.000 \\
\hline $\mathrm{C}_{4} \mathrm{H}_{2}$ & 1 & 357.000 & 5.180 & 0.000 & 0.000 & 1.000 \\
\hline $\mathrm{C}_{4} \mathrm{H}_{2} \mathrm{OH}$ & 2 & 224.700 & 4.162 & 0.000 & 0.000 & 1.000 \\
\hline $\mathrm{C}_{4} \mathrm{H}_{8}$ & 2 & 357.000 & 5.176 & 0.000 & 0.000 & 1.000 \\
\hline $\mathrm{C}_{4} \mathrm{H}_{9}$ & 2 & 357.000 & 5.176 & 0.000 & 0.000 & 1.000 \\
\hline$s-\mathrm{C}_{4} \mathrm{H}_{9}$ & 2 & 357.000 & 5.176 & 0.000 & 0.000 & 1.000 \\
\hline$i-\mathrm{C}_{4} \mathrm{H}_{9}$ & 2 & 357.000 & 5.176 & 0.000 & 0.000 & 1.000 \\
\hline $\mathrm{C}_{5} \mathrm{H}_{2}$ & 1 & 357.000 & 5.180 & 0.000 & 0.000 & 1.000 \\
\hline $\mathrm{C}_{5} \mathrm{H}_{3}$ & 1 & 357.000 & 5.180 & 0.000 & 0.000 & 1.000 \\
\hline $\mathrm{C}_{6} \mathrm{H}_{2}$ & 1 & 357.000 & 5.180 & 0.000 & 0.000 & 1.000 \\
\hline $\mathrm{C}_{6} \mathrm{H}_{5}$ & 2 & 412.300 & 5.349 & 0.000 & 0.000 & 1.000 \\
\hline $\mathrm{C}_{6} \mathrm{H}_{5}(\mathrm{~L})$ & 2 & 412.300 & 5.349 & 0.000 & 0.000 & 1.000 \\
\hline $\mathrm{C}_{6} \mathrm{H}_{5} \mathrm{O}$ & 2 & 450.000 & 5.500 & 0.000 & 0.000 & 1.000 \\
\hline $\mathrm{C}_{5} \mathrm{H}_{5} \mathrm{OH}$ & 2 & 450.000 & 5.500 & 0.000 & 0.000 & 1.000 \\
\hline $\mathrm{C}_{6} \mathrm{H}_{6}$ & 2 & 412.300 & 5.349 & 0.000 & 0.000 & 1.000 \\
\hline $\mathrm{C}_{6} \mathrm{H}_{6}$ & 2 & 464.800 & 5.290 & 0.000 & 10.30 & 1.000 \\
\hline $\mathrm{C}_{6} \mathrm{H}_{7}$ & 2 & 412.300 & 5.349 & 0.000 & 0.000 & 1.000 \\
\hline $\mathrm{CH}_{2} \mathrm{CO}$ & 2 & 436.000 & 3.970 & 0.000 & 0.000 & 2.000 \\
\hline $\mathrm{CH}_{3} \mathrm{CC}$ & 2 & 252.000 & 4.760 & 0.000 & 0.000 & 1.000 \\
\hline $\mathrm{CH}_{3} \mathrm{CO}$ & 2 & 436.000 & 3.970 & 0.000 & 0.000 & 2.000 \\
\hline $\mathrm{CH}_{3} \mathrm{O}$ & 2 & 417.000 & 3.690 & 1.700 & 0.000 & 2.000 \\
\hline $\mathrm{CH}_{3} \mathrm{OH}$ & 2 & 481.800 & 3.626 & 0.000 & 0.000 & 1.000 \\
\hline $\mathrm{CH}_{4} \mathrm{O}$ & 2 & 417.000 & 3.690 & 1.700 & 0.000 & 2.000 \\
\hline CNC & 1 & 232.400 & 3.828 & 0.000 & 0.000 & 1.000 \\
\hline CNN & 1 & 232.400 & 3.828 & 0.000 & 0.000 & 1.000 \\
\hline $\mathrm{CH}_{2} \mathrm{CHCCH}$ & 2 & 357.000 & 5.180 & 0.000 & 0.000 & 1.000 \\
\hline $\mathrm{CH}_{2} \mathrm{CHCCH}_{2}$ & 2 & 357.000 & 5.180 & 0.000 & 0.000 & 1.000 \\
\hline $\mathrm{CH}_{2} \mathrm{CHCH}_{2}$ & 2 & 260.000 & 4.850 & 0.000 & 0.000 & 1.000 \\
\hline $\mathrm{CH}_{2} \mathrm{CHCHCH}$ & 2 & 357.000 & 5.180 & 0.000 & 0.000 & 1.000 \\
\hline $\mathrm{CH}_{2} \mathrm{CHCHCH}_{2}$ & 2 & 357.000 & 5.180 & 0.000 & 0.000 & 1.000 \\
\hline $\mathrm{CH}_{3} \mathrm{CCCH}_{2}$ & 2 & 357.000 & 5.180 & 0.000 & 0.000 & 1.000 \\
\hline $\mathrm{CH}_{3} \mathrm{CCCH}_{3}$ & 2 & 357.000 & 5.180 & 0.000 & 0.000 & 1.000 \\
\hline $\mathrm{CH}_{3} \mathrm{CCH}_{2}$ & 2 & 260.000 & 4.850 & 0.000 & 0.000 & 1.000 \\
\hline $\mathrm{CH}_{3} \mathrm{CHCH}$ & 2 & 260.000 & 4.850 & 0.000 & 0.000 & 1.000 \\
\hline $\mathrm{CH}_{3} \mathrm{CH}_{2} \mathrm{CCH}$ & 2 & 357.000 & 5.180 & 0.000 & 0.000 & 1.000 \\
\hline $\mathrm{CH}_{3} \mathrm{CHO}$ & 2 & 436.000 & 3.970 & 0.000 & 0.000 & 2.000 \\
\hline $\mathrm{H}_{2} \mathrm{C}_{4} \mathrm{O}$ & 2 & 357.000 & 5.180 & 0.000 & 0.000 & 1.000 \\
\hline $\mathrm{H}_{2} \mathrm{CCCCH}$ & 2 & 357.000 & 5.180 & 0.000 & 0.000 & 1.000 \\
\hline $\mathrm{H}_{2} \mathrm{CCCCH}_{2}$ & 2 & 357.000 & 5.180 & 0.000 & 0.000 & 1.000 \\
\hline $\mathrm{H}_{2} \mathrm{CCCH}$ & 2 & 252.000 & 4.760 & 0.000 & 0.000 & 1.000 \\
\hline
\end{tabular}


Table 3. Continued.

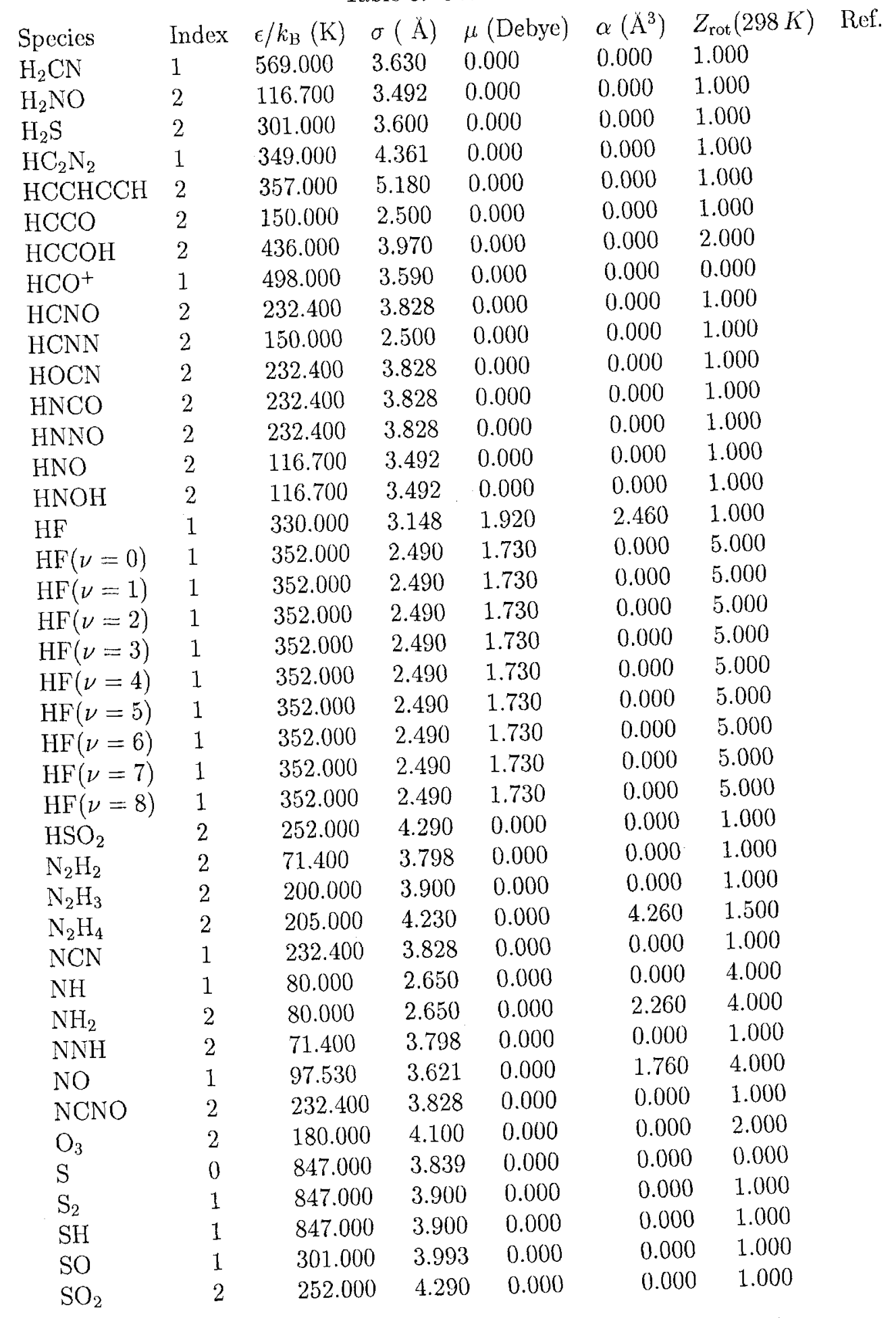


Table 3. Continued.

\begin{tabular}{|c|c|c|c|c|c|c|c|}
\hline Spccies & Index & $\epsilon / k_{\mathrm{B}}(\mathrm{K})$ & $\sigma(\AA)$ & $\mu$ (Debye) & $\alpha\left(\AA^{3}\right)$ & $Z_{\text {rot }}(298 K)$ & Ref. \\
\hline $\mathrm{SO}_{3}$ & 2 & 378.400 & 4.175 & 0.000 & 0.000 & 1.000 & \\
\hline $\mathrm{SiH}_{4}$ & 2 & 207.600 & 4.084 & 0.000 & 0.000 & 1.000 & \\
\hline $\mathrm{SiH}_{3}$ & 2 & 170.300 & 3.943 & 0.000 & 0.000 & 1.000 & \\
\hline $\mathrm{SiH}_{2}$ & 2 & 133.100 & 3.803 & 0.000 & 0.000 & 1.000 & \\
\hline $\mathrm{SiH}$ & 1 & 95.800 & 3.662 & 0.000 & 0.000 & 1.000 & \\
\hline $\mathrm{Si}$ & 0 & 3036.00 & 2.910 & 0.000 & 0.000 & 0.000 & \\
\hline $\mathrm{Si}_{2} \mathrm{H}_{6}$ & 2 & 301.300 & 4.828 & 0.000 & 0.000 & 1.000 & \\
\hline $\mathrm{Si}_{2} \mathrm{H}_{5}$ & 2 & 306.900 & 4.717 & 0.000 & 0.000 & 1.000 & \\
\hline $\mathrm{Si}_{2} \mathrm{H}_{4}$ & 2 & 312.600 & 4.601 & 0.000 & 0.000 & 1.000 & \\
\hline $\mathrm{Si}_{2} \mathrm{H}_{3}$ & 2 & 318.200 & 4.494 & 0.000 & 0.000 & 1.000 & \\
\hline $\mathrm{Si}_{2} \mathrm{H}_{2}$ & 2 & 323.800 & 4.383 & 0.000 & 0.000 & 1.000 & \\
\hline $\mathrm{Si}_{2}$ & 1 & 3036.00 & 3.280 & 0.000 & 0.000 & 1.000 & \\
\hline $\mathrm{Si}_{3}$ & 2 & 3036.00 & 3.550 & 0.000 & 0.000 & 1.000 & \\
\hline $\mathrm{SiF}_{4}$ & 2 & 171.900 & 4.880 & 0.000 & 0.000 & 1.000 & \\
\hline $\mathrm{H}_{2} \mathrm{SiSiH}_{2}$ & 2 & 312.600 & 4.601 & 0.000 & 0.000 & 1.000 & \\
\hline $\mathrm{H}_{3} \mathrm{SiSiH}$ & 2 & 312.600 & 4.601 & 0.000 & 0.000 & 1.000 & \\
\hline $\mathrm{Si}_{3} \mathrm{H}_{8}$ & 2 & 331.200 & 5.562 & 0.000 & 0.000 & 1.000 & \\
\hline $\mathrm{e}^{-}$ & 0 & 850.000 & 425.0 & 0.000 & 0.000 & 1.000 & \\
\hline $\mathrm{AsH}_{3}$ & 2 & 259.800 & 4.145 & 0.000 & 0.000 & 1.000 & \\
\hline $\mathrm{As}_{2}$ & 1 & 1045.50 & 5.510 & 0.000 & 0.000 & 1.000 & \\
\hline $\mathrm{GaMe}_{3}$ & 2 & 378.200 & 5.520 & 0.000 & 0.000 & 1.000 & \\
\hline $\mathrm{GaMe}_{2}$ & 2 & 675.800 & 5.220 & 0.000 & 0.000 & 1.000 & \\
\hline GaMe & 2 & 972.700 & 4.920 & 0.000 & 0.000 & 1.000 & \\
\hline $\mathrm{Ga}$ & 0 & 2961.80 & 4.620 & 0.000 & 0.000 & 0.000 & \\
\hline $\mathrm{Br}_{2}$ & 1 & 520.000 & 4.268 & - & - & & {$[56]$} \\
\hline $\mathrm{SF}_{6}$ & 2 & 207.700 & 5.252 & - & - & & [54] \\
\hline $\mathrm{Cl}$ & 0 & 130.800 & 3.613 & 0.000 & 0.000 & 1.000 & \\
\hline $\mathrm{Cl}^{-}$ & 0 & 130.800 & 3.613 & 0.000 & 0.000 & 1.000 & \\
\hline $\mathrm{HCl}$ & 1 & 344.700 & 3.339 & 1.084 & 0.000 & 1.000 & \\
\hline $\mathrm{F}$ & 0 & 80.000 & 2.750 & 0.000 & 0.000 & 0.000 & \\
\hline $\mathrm{F}_{2}$ & 1 & 125.700 & 3.301 & 0.000 & 1.600 & 3.800 & \\
\hline K & 0 & 850.000 & 4.250 & 0.000 & 0.000 & 1.000 & \\
\hline $\mathrm{KO}$ & 1 & 383.000 & 3.812 & 0.000 & 0.000 & 1.000 & \\
\hline $\mathrm{KOH}$ & 2 & 1213.00 & 4.520 & 0.000 & 0.000 & 1.000 & \\
\hline $\mathrm{KO}_{2}$ & 2 & 1213.00 & 4.690 & 0.000 & 0.000 & 1.000 & \\
\hline KH & 1 & 93.300 & 3.542 & 0.000 & 0.000 & 1.000 & \\
\hline $\mathrm{K}^{+}$ & 0 & 850.000 & 4.250 & 0.000 & 0.000 & 1.000 & \\
\hline $\mathrm{KCl}$ & 1 & 1989.00 & 4.186 & 0.000 & 0.000 & 1.000 & \\
\hline
\end{tabular}

